Revista de Psicología y Educación / Journal of Psychology and Education, 2022, 17(1), 97-115 (www.rpye.es) Doi: https://doi.org/10.23923/rpye2022.01.219

ISSN: $1699-9517$

\title{
Situaciones vitales estresantes y sintomatología depresiva en adolescentes rurales
}

\author{
Morelia Erandeni Gil-Díaz, María Elena Rivera-Heredia* y \\ María de Lourdes Vargas-Garduño \\ Universidad Michoacana de San Nicolás de Hidalgo
}

\begin{abstract}
Resumen: Ante el incremento de la sintomatología depresiva en población adolescente se requiere profundizar el conocimiento sobre las situaciones que ésta vive, según cada contexto y grupo sociocultural. El presente estudio se enfocó en evaluar situaciones vitales estresantes y sintomatología depresiva en 105 adolescentes de bachillerato de una comunidad rural del estado de Michoacán en México, de los cuales el 35,2\% fueron hombres y $64,8 \%$ fueron mujeres, de entre 15 y 17 años (M $=15,52 ; D E=.557)$. El método fue cuantitativo y transversal. Se evaluaron situaciones estresantes, sintomatología depresiva e indicadores de riesgo en la trayectoria académica. Entre los principales resultados se encontró que los sucesos de vida estresantes que correlacionaron con un mayor número de síntomas depresivos fueron las dificultades de tipo amoroso, las discusiones con la pareja y el divorcio de los padres. Las mujeres presentaron puntuaciones más altas que los hombres en la mayoría de los síntomas depresivos. Se concluye que la presencia de ciertos eventos estresantes en la vida de los adolescentes se relacionan con el desarrollo de síntomas depresivos. Se describen algunas temáticas que podrían retomarse en programas de orientación para adolescentes de contextos rurales, siendo el escenario educativo un espacio ideal para su aplicación.

Palabras clave: Estudiantes, Depresión, Estrés, Adolescentes, México
\end{abstract}

\section{Stressing life situations and depressive symptomatology in rural adolescents}

\begin{abstract}
Given the increase in depressive symptoms in adolescents, researchers must deepen knowledge about the situations associated with the development of such symptoms. These are different in each context and sociocultural group. The present study focused on evaluating stressful life situations and depressive symptoms in 105 high school students from a rural community in the State of Michoacán, Mexico. Of them, $35.2 \%$ were male and $64.8 \%$ were female, aged between 15 and 17 years old $(M=$ $15.52, S D=.557)$. a quantitative and cross-sectional method was used. Stressful situations, depressive symptoms, and risk indicators were evaluated across the academic trajectory. The main results showed that the stressful life events that correlated with a greater number of depressive symptoms were love issues and arguments with the partner. Higher scores for depressive symptoms were also observed in females than in males. It is concluded that the presence of certain stressful events in adolescents' life is related to the development of depressive symptoms. In the present study, some themes that could be taken up for intervention strategies were identified, and the educational context an ideal space in which they could be implemented.
\end{abstract}

Keyword: Students, Depression, Stress, Adolescents, México.

La presencia de sintomatología depresiva es frecuente en todo el mundo, llegando a afectar aproximadamente a 350 millones de personas. Puede ocasionar conflictos en la vida de los individuos a nivel laboral,

Recibido: 05/09/2021 - Aceptado: 14/01/2022 - Avance online: 20/01/2022

*Correspondencia: María Elena Rivera-Heredia

Universidad Michoacana de San Nicolás de Hidalgo.

Dirección: 58110 , Morelia, México.

E-mail: maelenarivera@gmail.com

Gil-Díaz, M. E., Rivera-Heredia, M. E. y Vargas-Garduño, M. L. (2022). Situaciones Gil-Díaz, M. E., Rivera-Heredia, M. E. Y Vargas-Garduño, M. L. (2022). Situaciones Psicología y Educación, 17(1), 97-115, https://doi.org/10.23923/rpye2022.01.219 familiar y social, especialmente cuando es de larga duración y con gran intensidad (Organización Mundial de la Salud [OMS], 2016). Al ubicar la depresión en adolescentes es necesario considerar las implicaciones que esta llega a tener en cuanto a temas escolares - relacionados con el desempeño escolar (Rahman et al., 2018). 
Al hablar de depresión, es importante mencionar la sintomatología depresiva, la cual se puede definir como el conjunto de síntomas característicos de la depresión que llega a producir dificultades o complicaciones en la vida cotidiana de las personas los cuales son: irritabilidad, disforia, anhedonia, agitación, dificultad para concentrarse, insomnio o dormir en exceso, culpa, ideación suicida, dificultad para disfrutar actividades, fatiga, pérdida o aumento del apetito (Chapí, 2013).

De acuerdo con Rivera-Rivera et al. (2015) la depresión y su sintomatología son consideradas como un problema de salud pública a nivel mundial, ya que sus consecuencias impactan en diversas áreas de la vida. Estas pueden llegar a ser el bajo rendimiento escolar o el abuso de sustancias, entre otras. Es importante detectar sentimientos de tristeza presentes por un período extenso de tiempo, como también, la incapacidad para realizar actividades de la vida cotidiana o una afectación a la calidad de vida de los jóvenes. En este mismo sentido, la existencia de sintomatología depresiva en los adolescentes tiene como consecuencia una alteración en su proceso de desarrollo y puede causar afectaciones en el funcionamiento de la persona, incluso en el ámbito académico (López-López et al., 2021).

De acuerdo con un estudio realizado por Rahman et al. (2018) la presencia de sintomatología depresiva puede impactar en el logro académico de los estudiantes, lo cual puede verse reflejado en las bajas calificaciones. En este mismo sentido Cattelino et al. (2020) y Diaconu-Gherasim y Măirean (2020), señalan la relación entre la presencia de sintomatología depresiva y un desempeño inadecuado, así como con diferentes tipos de problemáticas en el desempeño escolar, el cual ocasiona en el alumno una lucha constante para la realización de las tareas académicas. De igual forma, estos autores coinciden en que esta situación afecta la autoconfianza de los adolescentes para realizar las diferentes tareas académicas relacionadas con el desempeño académico. Además también se registran problemas para interactuar con los compañeros, lo cual afecta el desempeño y el éxito de los adolescentes en la escuela y tiene implicaciones en su formación y en la adquisición del conocimiento. Ante estas situaciones Rahman et al. (2018) señalan la importancia de aumentar las redes de apoyo y la conexión social en las aulas.

De este modo, se observa que aquellos adolescentes que reportaban mayor sintomatología depresiva, presentan problemas en cuanto a su desempeño académico y también un pobre o deficiente involucramiento en las cuestiones escolares (Chow et al., 2015; Froid et al., 2008; Humenskyetal., 2010). En estas investigaciones además de resaltar la importancia de realizar intervenciones centradas en disminuir la presencia de sintomatología depresiva, se hace énfasis en el rol fundamental que tiene el personal que labora en las escuelas desde los profesores, hasta el personal administrativo y los directores en cuanto a brindar un manejo adecuado de los casos con adolescentes que presenten situaciones relacionadas con la salud mental.

Es así que, estas investigaciones centradas en la presencia de sintomatología depresiva y el aprovechamiento o desempeño escolar, muestran una área de oportunidad que debe ser fortalecida desde el campo de la prevención, ya que además de verse afectadas las cuestiones académicas, también se impacta la calidad de vida y el bienestar de los adolescentes, sobre todo al considerar que la presencia de sintomatología depresiva se encuentra relacionada con las conductas suicidas.

Aunque la sintomatología depresiva puede estar presente a cualquier edad, para la realización de este trabajo únicamente se enfocó en el estudio de la población adolescente, para lo cual se retomaron algunos estudios que han recuperado información relevante sobre la sintomatología depresiva estudiando solo a dicha población. Estos estudios 
se han centrado en describir las características de la depresión en la adolescencia (Chapí, 2013; Li at al., 2019). Mientras que otras investigaciones se han centrado en describir las sintomatología depresiva en adolescentes escolarizados (Andrade-Palos et al., 2012; Fonseca-Pedrero et al., 2011; Ghosh et al., 2019; OspinaOspina et al., 2011 ; Veytia-López, et al., 2016). Otros estudios han abordado la sintomatología depresiva y los factores que influyen en su desarrollo (Rivera-Heredia et al., 2013). Por otro lado, también existe información sobre la influencia que pueden llegar a tener las variables familiares en el desarrollo de esta sintomatología (Rivera-Rivera et al., 2015). Estas investigaciones han resaltado incluso que la existencia de sintomatología depresiva es cada vez más frecuente en esta población. Además, también se tiene registrado que aquellas personas que presentaron un estado depresivo mayor en la adolescencia, tienen mayores posibilidades de presentarlo en la etapa adulta (González-Forteza, et al., 2015; Jiménez-Tapia, et al., 2015).

Para profundizar en la comprensión de qué ocurre con la población adolescentes y la sintomatología depresiva se han identificado diversos factores asociados a la misma, entre ellos el género, encontrándose diferencias estadísticamente significativas en la presencia de sintomatología depresiva entre hombres y mujeres (Duggal, et al., 2002; Khesht-Masjedi, et al., 2017; Nunes et al., 2017). De igual forma, en un estudio de Granrud et al. (2019) las mujeres reportaron con mayor frecuencia síntomas depresivos, tales como desesperanza, problemas con el sueño, sentirse tristes, tensos y preocuparse demasiado por las cosas o situaciones. En México, González-Forteza et al. (2015) destacan que la prevalencia de estados depresivos en población joven ha ido en aumento, específicamente los jóvenes que se encuentran en zonas socio-urbanas conflictivas, siendo las mujeres quienes presentan mayor sintomatología depresiva.

En este mismo orden de ideas, Buitrago et al. (2017), Rivera et al. (2018) y Tafur-Orahlio (2020), señalan como factor asociado a la presencia de sintomatología depresiva, variables relacionadas con el ambiente familiar, tales como cuadros depresivos en la madre y la ausencia de un ambiente familiar que brinde cuidado y apoyo al adolescente.

También existen estudios que hacen referencia a las diferencias en relación a la edad, como en el caso de Fonseca-Pedrero et al. (2011) quienes describen que en el rango de 14 a 16 años de edad se registra una mayor presencia de sintomatología depresiva. Este estudio sugiere la importancia de realizar un diagnóstico y tratamiento adecuados. Se resalta la relevancia de identificar los factores de riesgo y protección para los adolescentes. Así, dichos autores junto con Rivera-Heredia et al. (2013) mencionan que la sintomatología depresiva se encuentra asociada a diversos factores, algunos de ellos son: baja autoestima, consumo de tabaco, alcohol y otras drogas, violencia intrafamiliar, baja comunicación entre padres e hijos, las prácticas parentales, dificultades en el manejo de la tristeza, el manejo del enojo, la incapacidad para buscar ayuda y la migración de la madre.

Un conjunto de factores de riesgo también importantes para la presencia de sintomatología depresiva son los sucesos estresantes de vida, debido al impacto que pueden llegar a tener estos en las vidas de las personas. Es así que, se tiene registro de la influencia de sucesos de tipo estresante en el desarrollo e inicio de síntomas de depresión (Pérez-Padilla et al., 2017). Los sucesos vitales estresantes se pueden definir como aquellos hechos que las personas perciben como relevantes en sus vidas y que tuvieron un fuerte impacto en su cambio evolutivo. Por lo que, para que dichos eventos sean considerados como estresantes es necesario que: sean vistos como negativos o indeseables y que produzcan un cambio vital en la vida de las personas (Suárez, 2010).

Así, algunos estudios señalan (González et al., 2018; Pascoe et al., 2020; Shapero et al., 2014) que la presencia de eventos estresantes tanto en adultos como en adolescentes, 
además de aumentar la aparición de síntomas depresivos, contribuyen a la aparición o desarrollo de un estado depresivo mayor. Por lo que, los eventos estresantes pueden llegar a originarse desde distintas situaciones de lo familiar, escolar o personal, pero su efecto es tal, que pueden alterar la normalidad de la vida de las personas. En este mismo tema Veytia-López et al. (2012) encontraron que la intensidad de los eventos estresantes reportados por los adolescentes era predictora de la presencia de síntomas depresivos.

También, Ferreira et al. (2012) señalan que la presencia de eventos vitales negativos aumenta la sintomatología depresiva, mientras que los eventos vitales positivos la disminuyen. Por otro lado, Stilkkelbrock et al. (2016) estudiaron el papel de la desregulación emocional, los eventos vitales negativos (perder a un ser querido, problemas de salud y dificultades interpersonales) y los síntomas depresivos. Mientras que en otros estudios (Aliri et al., 2019; Askeland et al., 2020; McMahon et al., 2020) identificaron cómo la presencia de situaciones estresantes de tipo familiar, de pareja, escolar y de salud están asociadas con la presencia y el aumento de sintomatología depresiva. Recordando que durante la etapa de la adolescencia pueden existir otros eventos estresantes relacionados con pérdidas, problemas de salud y cambios en las relaciones interpersonales. De igual forma, Yue et al. (2016) resaltan la relación que existe entre los eventos o situaciones estresantes y la presencia e intensidad de la sintomatología depresiva; además, estos autores también mencionan la influencia que puede llegar a tener el contexto cultural en el desarrollo de los síntomas depresivos.

Dentro de este mismo tema, al hablar de eventos estresantes autores como Barcelata et al. (2012) señalan que el impacto de los eventos estresantes también dependerá de diversos aspectos como la cultura, el género y la posición socioeconómica de los adolescentes. Por otro lado, cabe mencionar que la presencia de los eventos estresantes está asociada a la presencia o el desarrollo de diversas problemáticas, tales como el consumo excesivo de alcohol y tabaco en adolescentes, entre otras dificultades de comportamiento (Villegas-Pantoja et al., 2014).

De esta forma, una gran mayoría de los estudios revisados que informan sobre la presencia de eventos vitales estresantes y sintomatología depresiva en adolescentes se han realizado en contextos urbanos, por lo que se considera relevante generar mayor conocimiento sobre este grupo poblacional en zonas rurales, que contribya a comprender cómo es que los adolescentes en estas zonas se ven afectados, para tener con ello un panorama más completo en lo referente a la sintomatología depresiva en adolescentes que asisten a la escuela.

Por todo ello, los objetivos de la presente investigación fueron: 1) identificar la sintomatología depresiva y los sucesos vitales estresantes presentados con mayor frecuencia en los participantes, 2) conocer las diferencias de género en cuanto a sintomatología depresiva y sucesos estresantes, para finalmente, 3) analizar la relación existente entre las situaciones estresantes y la sintomatología depresiva; todo ello en una muestra de adolescentes, del nivel medio superior de una zona rural del estado de Michoacán en México.

\section{MÉTODO}

\section{PARTICIPANTES}

Para el presente estudio se contó con la participación de 105 adolescentes, alumnos de educación media superior de una institución educativa ubicada en una zona rural que además de ofertar los estudios a nivel bachillerato, al mismo tiempo cuenta con la opción de tener una formación como técnico profesional agropecuario. Todos los adolescentes participantes estaban inscritos en el segundo año de bachillerato, encontrándose un $35,2 \%$ de hombres (n $=21)$ y un $64,8 \%$ de mujeres $(n=55)$, de entre 15 y 17 años, de los cuales un $52,8 \%$ 
contaban con 15 años de edad $(M=15,52$ y una $D E=.56)$. El $66,1 \%$ de los participantes vivían en la localidad de Maravatío y un 6,6\% en la de Tungareo, mientras que el resto de los participantes $(27,3 \%)$ radicaba en distintas comunidades rurales circundantes, todavía más pequeñas.

Es importante mencionar que los directivos de la institución seleccionaron a los grupos con los cuales se trabajaría. Por lo que los participantes fueron alumnos perteneciente a tres grupos, todos del segundo grado. Para invitarlos a participar se realizó una conferencia sobre recursos psicológicos y prevención del suicidio a la cual también se invitó a los padres de familia. Al terminar la conferencia se comentó que se estarían aplicando algunos instrumentos para recuperar información sobre la salud mental de los estudiantes. Junto con las autoridades educativas se designó un día para la aplicación de los instrumentos; el director del plantel se encargó de avisar a los alumnos para que estuvieran presentes ese día. El momento de la aplicación de consentimientos informados y de los instrumentos se realizó en las primeras horas del día, durante el espacio de las tutorías, dentro del aula.

\section{INSTRUMENTOS DE MEDIDA}

Para evaluar la presencia de sintomatología depresiva se utilizó la Escala del Centro de Estudios Epidemiológicos CESD de Radloff (1977), específicamente se aplicó para este estudio la versión revisada (CESD-R) validada en México por Reyes Ortega et al. (2003) y adaptada para adolescentes por GonzálezForteza et al. (2008), la cual es una prueba de tamizaje que evalúa la presencia de sintomatología depresiva en las últimas dos semanas y consta de 35 reactivos a los que se incorporaron 4 reactivos de la Escala de Roberts (1980), que miden ideación suicida, quedando un total de 39 reactivos. Las opciones de respuesta son del 0 al 4 , donde 0 corresponde a escasamente ( 0 a 1 días) y 4 (casi diario durante las últimas dos semanas, de 10 a 14 días). De este modo, la versión revisada de la escala CESD-R quedó conformada con subdimensiones que evalúan: Disforia/Estado de Ánimo Deprimido, Cambios Drásticos en el Peso, Tipo de Pensamiento, Agitación - Retardo Psicomotor, Ideación Suicida, Problemas del Sueño, Amigos/Ocupación/ Familia, Culpa Excesiva o Inapropiada, y Anhedonia/Disminución del Placer o interés en las actividades (Rivera-Heredia \& Hermosillo de la Torre, 2019). La escala cuenta con una confiabilidad de alpha de Cronbach de .93 de acuerdo con lo señalado por GonzálezForteza et al. (2008).

El segundo instrumento que se utilizó fue la Escala de Reajuste Social (SRRS), de Holmes y Rahe (1967), la cual explora la presencia o vivencia de 43 eventos estresantes durante el último año. Se aplicó la versión adaptada al español por Bruner et al. (1994) y confirmada 16 años después por Acuña et al. (2012), la cual tiene una correlación entre jueces de entre, 72 y ,90. Para la calificación, los autores asignaron de manera predeterminada y validada por jueces, una puntuación para cada suceso, de modo que se suman dichas puntuaciones y si el resultado se encuentra por debajo de 150 corresponde a un nivel de estrés bajo, en caso que la puntuación sume 300 puntos se considera un nivel elevado que puede afectar la salud. Para el presente estudio se utilizaron las puntuaciones de cada suceso sin tomar en cuenta la clasificación del nivel de estrés. Además también se identificaron cuáles de los 43 sucesos estresantes fueron experimentados por los participantes con mayor frecuencia.

Finalmente, el tercer instrumento utilizado fue el Cuestionario para medir Indicadores de Riesgo en la Trayectoria Académica (PérezPadilla et al., 2021). Este instrumento se utilizó con el objetivo de evaluar otros sucesos estresantes que pueden interferir en el curso de los estudios, y por tanto, estar asociados con la deserción o la reprobación. Se utilizaron 12 de los 17 reactivos. Las opciones de respuesta son $\mathrm{SI}$ o NO (con un nivel de medición nominal). Evalúa diferentes situaciones de la vida que pueden considerarse como estresantes 
cuya presencia puede incrementar el riesgo de la continuidad de los estudios. El nivel de análisis de este cuestionario es por reactivo y no a manera de escala, por lo que no se reporta la confiabilidad del mismo. Cada suceso se analizó como variable independiendente contrastando la sintomatología depresiva entre quienes tuvieron o no cada uno de estos sucesos. Las preguntas corresponden a: 1) área familiar (¿̇Vive tu madre? 2 ¿Vive tu padre? padres viven juntos?, ¿̇ienes hijos?, Además de estudiar ¿̇tienes otras obligaciones como cuidar a tus hijos, a tus padres, o hermanos?, ¿Vives con tu familia en la ciudad en donde estudias?); 2) al área escolar ¿̇Tienes asignaturas pendientes que tienes que volver a cursar?, ¿̇ Has tenido algún problema con tus compañeros de clase?, ¿Has tenido algún problema con tus profesores? y ¿̇Has tenido algún problema con tus directivos de la escuela?. Y por último, 3) el área psicosocial (¿̇Tienes que compaginar estudios y trabajo?, ¿̇Dedicas más de una hora en transportarte de tu casa a la escuela?, ¿Has tenido dificultades económicas?, ¿Has tenido dificultades de tipo amoroso?, ¿̇Has vivido alguna situación de violencia?, ¿Has tenido algún problema de salud?).

\section{PROCEDIMIENTO}

Se contactó con los directivos de la institución y se solicitó el permiso para trabajar con sus alumnos. Se obtuvo la autorización de los directivos y una vez que se designaron los grupos con los cuales se trabajaría, se realizó una junta con los padres de familia para solicitar la autorización e informarlos sobre el trabajo que se realizaría, además en dicha reunión se informó sobre la importancia de la salud mental en los adolescentes. A los alumnos se les proporcionó una breve explicación sobre lo que se trabajaría y se les pidió que firmaran un consentimiento informado en el cual se les aseguraba que la información era confidencial. La aplicación de los instrumentos se realizó en una sola sesión, con una duración de una hora, en el aula de cada grupo. Así la aplicación fue de manera autoaplicable con un formato de lápiz-papel y fue supervisada por la investigadora principal del proyecto.

\section{ANÁLISIS DE DATOS}

Se realizaron análisis estadísticos de tipo descriptivo, tales como frecuencias y porcentajes para identificar la frecuencia de los sucesos vitales estresantes y la sintomatología depresiva que presentan los adolescentes. En cuanto a la comparación de la sintomatología depresiva y sucesos estresantes entre hombres y mujeres, se realizaron pruebas de comparación de medias con la prueba $t$ de student. Y finalmente se utilizó la prueba Chi cuadrada para comparar la frecuencia de aparición de los indicadores de riesgo de la trayectora académica entre hombres y mujeres. Asimismo, para evaluar la relación existente entre las situaciones estresantes y la sintomatología depresiva se aplicó el coeficiente de correlación de Spearman (se utilizó este estadístico, debido a que el nivel de medición del cuestionario de indicadores de riesgo en la trayectoria académica era nominal). Todos los análisis estadísticos realizados fueron con el apoyo del programa estadístico SPSS 20.

\section{RESULTADOS}

A continuación, se presentan los principales resultados obtenidos en este estudio. Para comenzar se identificó la sintomatología depresiva en los participantes (ver Tabla 1), cuyas puntuaciones promedio variaron de ,3 para el caso de la Ideación Suicida hasta 1,3 , el cual fue el promedio más alto y se encontró en la subdimensión de Disforia. En general, la puntuación promedio de todas las subdimensiones se ubicó en niveles intermedios, pues corresponden a presentar síntomas depresivos de 3 a 4 días como máximo en las últimas 2 semanas.

Entre los 20 sucesos estresantes que los adolescentes mencionaron con mayor frecuencia se encontraron las navidades $(F q=37 ; 35,92 \%)$, la muerte de un familiar cercano $(F q=35 ; 33.98 \%)$, las vacaciones $(F q=32 ; 31,02 \%)$ y el inicio o término del ciclo escolar ( $F q=31 ; 30,10 \%)$. En la Tabla 2 puede apreciarse a detalle cada uno de ellos. 


\section{Tabla 1}

Puntuaciones promedio de los síntomas depresivos

\begin{tabular}{|c|c|c|}
\hline Subescala & Media & D.E. \\
\hline Disforia & 1.30 & 0.67 \\
\hline Culpa & 1.29 & 0.83 \\
\hline Anhedonia & 1.29 & 0.51 \\
\hline Problemas interpersonales & 1.28 & 0.61 \\
\hline Fatiga & 1.08 & 0.86 \\
\hline Problemas del sueño & 1.02 & 0.89 \\
\hline Ideación suicida & 0.32 & 0.76 \\
\hline Nota: las puntuaciones promedio se ordenaron de manera descendente. & \\
\hline
\end{tabular}

\section{Tabla 2}

Lista de sucesos vitales estresantes que han vivido los adolescentes en el último año de vida

\begin{tabular}{|c|c|c|c|c|}
\hline Suceso vital estresante & Frecuencia & $\%$ & Media & $\mathrm{DE}$ \\
\hline 1. Navidades & 37 & 35,92 & 45 &, 500 \\
\hline 2. Muerte de un familiar cercano & 35 & 33,98 & .41 & 494 \\
\hline 3. Vacaciones & 32 & 31,07 & 38 & ,489 \\
\hline 4. Se inicia o termina el ciclo escolar & 31 & 30,10 & 36 & ,484 \\
\hline 5. Cambios en los hábitos personales & 28 & 27,18 & 32 & ,468 \\
\hline 6. Enfermedad de un pariente cercano & 26 & 25.24 & 30 & 459 \\
\hline 7. Cambio en la forma o frecuencia de las diversiones & 22 & 21,36 & 25 & 435 \\
\hline 8. Logros personales excepcionales & 22 & 21,36 & 25 & ,435 \\
\hline 9. Cambio en los hábitos de sueño & 22 & 21,36 & .25 & 435 \\
\hline 10. Cambio a una nueva escuela & 18 & 17.48 & 20 & 406 \\
\hline 11. Cambio en los hábitos alimenticios & 18 & 17.48 & 20 & ,404 \\
\hline 12. Cambios importantes a nivel económico & 17 & 16.50 &, 19 & 397 \\
\hline 13. Llegada de nuevos miembros a la familia & 14 & 13.59 &, 16 & 368 \\
\hline 14. Cambio en las actividades sociales & 14 & 13,59 &, 16 & 366 \\
\hline 15. Cambio en el número de reuniones familiares & 12 & 11.65 &, 13 & 343 \\
\hline 16. Enfermedad o incapacidad grave & 12 & 11.65 &, 13 & 343 \\
\hline 17. Discusiones con la pareja & 9 & 8.74 & 10 & 303 \\
\hline 18. Cambio de horario o condiciones de trabajo & 8 & 7.77 & 09 & 289 \\
\hline 19. Cambios importantes en las condiciones de vida & 8 & 7.77 & ,09 & 289 \\
\hline 20. Cambio de la frecuencia de las actividades religiosas & 8 & 7.77 & .09 & 288 \\
\hline
\end{tabular}

Nota: se presentan 20 de los 43 items de esta escala en función de la frecuencia con las que se mencionaron. 


\section{Tabla 3}

Sucesos vitales estresantes mencionados en el apartado de "otros problemas"

\begin{tabular}{|c|l|}
\hline & \multicolumn{1}{|c|}{ "Otros problemas" reportados por las y los participantes } \\
\hline 1 & Falta de comprensión de mi mamá, desconfianza \\
\hline 2 & Problemas en el desarrollo escolar \\
\hline 3 & Problemas familiares \\
\hline 4 & Muerte de una hermana \\
\hline 5 & Mi mamá sufrió una fractura y para mí fue difícil ayudarle y al mismo tiempo estudiar \\
\hline 6 & Tener a mi hijo y después perderlo \\
\hline 7 & Mi mamá estuvo enferma por algunos meses \\
\hline 8 & La muerte de mi abuelita \\
\hline 9 & Enamorarte de la persona que hace que descuides la escuela \\
\hline 10 & No me gusta la escuela a la que voy \\
\hline 11 & Extorsión telefónica \\
\hline
\end{tabular}

\section{Tabla 4}

Frecuencia y porcentaje de los indicadores de riesgo en la trayectoria académica

\begin{tabular}{|c|c|c|}
\hline Indicadores de Riesgo en la trayectoria académica & Si & No \\
\hline ¿Viven tus padres juntos? & $79(78,2 \%)$ & $22(21,8 \%)$ \\
\hline ¿Vives con tu familia en la ciudad donde estudias? & $77(73,3 \%)$ & $24(22,9 \%)$ \\
\hline Además de estudiar ¿tienes otras obligaciones como cuidar a tus padres, hermanos o hijos? & $22(21,8 \%)$ & $79(78,2 \%)$ \\
\hline ¿Tienes asignaturas pendientes que tienes que volver a cursar? & $22(2,0 \%)$ & $80(76,2 \%)$ \\
\hline ¿Dedicas más de una hora en transportarte de tu casa a la escuela? & $17(16,2 \%)$ & $85(81,0 \%)$ \\
\hline ¿Has tenido dificultades económicas que pongan en riesgo tus estudios? & $17(16,8 \%)$ & $84(83,2 \%)$ \\
\hline ¿Has tenido algún problema de salud que haya puesto en riesgo que continúes con tus estudios? & $16(15,7 \%)$ & $86(87,3 \%)$ \\
\hline ¿Has tenido dificultades de tipo amoroso que pongan en riesgo tus estudios? & $11(11 \%)$ & $89(89,0 \%)$ \\
\hline Nota: solo se incluyeron los factores de riesgo con porcentajes más altos y se presentan en orden decreciente. \\
\hline
\end{tabular}

Otro tipo de sucesos estresantes que se identificaron en este estudio, son los que tienen que ver con la separación de los padres, ya sea por no vivir en la misma ciudad o porque ellos tuvieron una ruptura conyugal, así como por sobrecarga de actividades en casa y dificultades económicas, además de problemas amorosos. Es importante mencionar que no se indagó el número de veces que los participantes experimentaron estos sucesos (ver Tabla 3).
Los indicadores de riesgo de la trayectoria escolar mencionados con mayor frecuencia por los adolescentes (ver Tabla 4) fueron el tener asignaturas pendientes por recursar $(22 \%)$, el tener que cuidar a sus padres, hermanos o hijos (22.8\%), el dedicar más de una hora de transporte entre la casa y la escuela $(16,2 \%)$ y el haber experimentado dificultades económicas (16,8\%). 
Continuando con los resultados relacionados con la presencia de situaciones estresantes en los adolescentes, también se exploró la diferencia en cuanto a los indicadores de riesgo que experimentaron los hombres y las mujeres encontrándose que al contrastarlos, en general el comportamiento es semejante, únicamente se encuentran diferencias estadísticamente significativas en: 1) Además de estudiar 2̇tienes otras obligaciones como cuidar a tus padres, hermanos o hijos?, en donde las mujeres mencionan en mayor porcentaje que los hombres si tenerlas $182 \%$ mujeres, $18 \%$ hombres (Chi cuadrada $=3,73$, $p=$,043), 2) ¿̇Tienes asignaturas pendientes que tienes que volver a cursar? encontrándose que un $59 \%$ de los hombres si han tenido asignaturas que han tenido que volver a cursar, mientras que las mujeres informan sólo un $41 \%$ (Chi cuadrada $=6,95$, p. $=$,009), 3) ¿̇Has tenido dificultades de tipo amoroso que pongan en riesgo tus estudios?, donde las mujeres mencionan sí haberlas tenido en un
$91 \%$ y los hombres en un $9 \%$ (Chi cuadrada $=$ $3,64, p=, 05)$.

Para darle seguimiento al objetivo de identificar las diferencias entre hombres y mujeres, en cuanto a la presencia de sintomatología depresiva se realizó una prueba t de student para muestras independientes. El análisis arrojó que existen diferencias estadísticamente significativas entre las dimensiones de Anhedonía, Problemas Del Sueño, Ideación Suicida, Fatiga y Culpa, pertenecientes a la escala de sintomatología depresiva CESD-R. Encontrándose que las mujeres obtuvieron puntuaciones significativas y más altas en comparación con los hombres en la mayoría de las subdimensiones (ver Tabla 5).

Finalmente, para conocer si la presencia de un indicador de riesgo influía en la aparición de un síntoma depresivo, se realizó una comparación utilizando la prueba estadística

\section{Tabla 5}

Comparación entre hombres y mujeres de las puntuaciones promedio en las dimensiones de sintomatología depresiva

\begin{tabular}{|c|c|c|c|c|c|c|c|}
\hline \multirow[b]{2}{*}{ Subescalas } & \multicolumn{2}{|c|}{ Hombres } & \multicolumn{2}{|c|}{ Mujeres } & \multirow[b]{2}{*}{$t$} & \multirow[b]{2}{*}{ gl } & \multirow[b]{2}{*}{$p$} \\
\hline & $M$ & $D E$ & $M$ & $D E$ & & & \\
\hline Anhedonia & 1,39 & 677 & 1,70 &, 654 & $-2,245$ & 103 &, 030 \\
\hline Problemas del sueño & ,72 & 669 & 1,33 & 1,038 & $-3,604$ & 103 & 001 \\
\hline Fatiga &, 82 & 658 & 1,22 &, 942 & $-2,509$ & 103 & 014 \\
\hline Culpa Excesiva & ,71 &, 565 & 1,13 & 873 & $-2,916$ & 103 &, 004 \\
\hline Ideación suicida &, 14 & ,373 & 42 & 891 & $-2,263$ & 103 & ,026 \\
\hline Amigos/ocupación/familia & 62 & 664 & 1,13 &, 958 & -3142 & 103 &, 001 \\
\hline Disforia & 46 &, 504 & 1,32 & 1.119 & $-5,301$ & 103 & ,001 \\
\hline
\end{tabular}


detde student. De acuerdo con esta prueba una proporción de los adolescentes que contaban con alguno de los indicadores de riesgo, también tenían la presencia de sintomatología depresiva. Al comparar las puntaciones en las diferentes dimensiones de sintomatología depresiva entre quienes han vivido $O$ no diferentes sucesos de vida estresantes (CESD $R)$, así como con los indicadores de riesgo de la trayectoria escolar, se encontró que los síntomas que se mencionaron de manera significativa en un mayor número de sucesos de vida estresantes fueron los Problemas de
Sueño, seguidos por la Anhedonia, la Fatiga y los Problemas Interpersonales, así como los siguientes indicadores de riesgo: las dificultades de tipo amoroso y el haber tenido alguna situación de violencia, las situaciones económicas que ponen en riesgo los estudios, la presencia de asignaturas pendientes por recursar, y el compaginar estudios con trabajo (ver Tabla 6). Llama la atención cómo las dificultades de tipo amoroso están presentes de manera significativa en las comparaciones realizadas con la mayoría de los síntomas depresivos.

\section{Tabla 6}

Comparación de las puntuaciones promedio en las dimensiones de sintomatología depresiva con diferentes indicadores de riesgo de la trayectoria escolar

\begin{tabular}{|c|c|c|c|c|c|c|c|c|c|}
\hline & & $n$ & 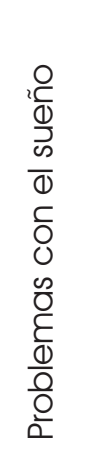 & 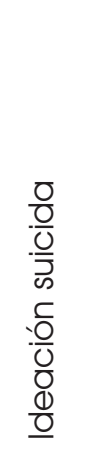 & $\begin{array}{l}\frac{0}{c} \\
\frac{0}{O} \\
\frac{\Phi}{c} \\
\frac{c}{\frac{1}{4}}\end{array}$ & $\begin{array}{l}0 \\
\frac{0}{0} \\
\frac{\bar{T}}{0} \\
\end{array}$ & 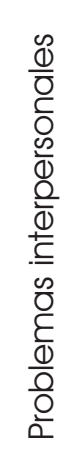 & $\begin{array}{l}\frac{0}{0} \\
\frac{0}{0} \\
\frac{\mathscr{D}}{0}\end{array}$ & $\frac{\delta}{\bar{J}}$ \\
\hline \multirow{3}{*}{$\begin{array}{l}\text { ¿Has tenido dificultades de } \\
\text { tipo amoroso que pongan } \\
\text { en riesgo tus estudios? }\end{array}$} & $\mathrm{Si}$ & 11 & 1,79 & .89 & 1,79 & .98 & 1,99 & 1,86 & 2,25 \\
\hline & No & 89 &, 98 & .25 & 1,22 & 1,85 & 1,18 & 1,22 & 1,18 \\
\hline & \multicolumn{2}{|c|}{$p$} &, $002^{*}$ & 148 & ,007* &, 01 * &, 001 * & ,003* &, 001 * \\
\hline \multirow{3}{*}{$\begin{array}{l}\text { ¿Has tenido alguna situación } \\
\text { de violencia que haya } \\
\text { puesto en riesgo el que } \\
\text { continúes tus estudios? }\end{array}$} & $\mathrm{Si}$ & 07 & 1,76 & 91 & 1,62 & 1,43 & 1,80 & 1,58 & 1,86 \\
\hline & No & 95 & 97 & 28 & 1,27 & 1,06 & 1,24 & 1,28 & 1,25 \\
\hline & \multicolumn{2}{|c|}{$p$} & ,020* & ,032* & 226 & , 128 & ,020* & 260 & 150 \\
\hline \multirow{3}{*}{$\begin{array}{l}\text { ¿Has tenido dificultades } \\
\text { económicas que pongan } \\
\text { en riesgo tus estudios? }\end{array}$} & $\mathrm{Si}$ & 17 & 1,65 & ,45 & 1,53 & 1,24 & 1,33 & 1,50 & 1,60 \\
\hline & No & 84 & 91 & ,30 & 1,24 & 1,06 & 1,27 & 1,26 & 1,24 \\
\hline & \multicolumn{2}{|c|}{$p$} & , 001 * & 479 &, $034^{*}$ & 453 & 685 & 174 &, 518 \\
\hline \multirow{3}{*}{$\begin{array}{c}\text { ¿Tienes asignaturas } \\
\text { pendientes que tienes que } \\
\text { volver a cursar? }\end{array}$} & $\mathrm{Si}$ & 22 & 1,20 & ,38 & 1,23 & ,76 & 1,71 & 1,15 & 1,27 \\
\hline & No & 80 & 98 & , 31 & 1,31 & 1,17 & 1,31 & 1,34 & 1,39 \\
\hline & \multicolumn{2}{|c|}{$\mathrm{p}$} & ,361 & 687 &, 536 &, $05^{\star}$ & ,356 & 283 &, 532 \\
\hline \multirow{3}{*}{$\begin{array}{c}\text { ¿Tienes que compaginar } \\
\text { estudios y trabajo? }\end{array}$} & $\mathrm{Si}$ & 06 & 39 & 17 & 1,30 & 1,17 & 1,26 & 1,38 & 1,33 \\
\hline & No & 92 & 1,06 & 33 & 1,38 & 1,10 & 1,29 & 1,31 & 1,31 \\
\hline & &, $006^{*}$ & 393 & 464 & ,918 & ,873 & 860 & 969 \\
\hline
\end{tabular}




\section{Tabla 7}

Correlaciones entre las dimensiones de sintomatología depresiva con los reactivos de las escalas de ajuste social y con los indicadores de riesgo a la trayectoria escolar

\begin{tabular}{|c|c|c|c|c|c|c|c|c|}
\hline 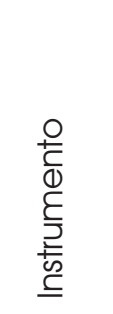 & Sucesos de vida estresantes & $\begin{array}{l}\frac{0}{\overline{0}} \\
\frac{0}{0} \\
\frac{-5}{0}\end{array}$ & 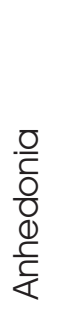 & 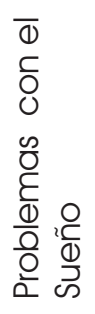 & $\begin{array}{l}\frac{0}{0} \\
\frac{0}{0} \\
\frac{7}{4}\end{array}$ & $\frac{\mathrm{O}}{\mathrm{O}}$ & 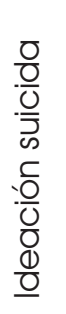 & 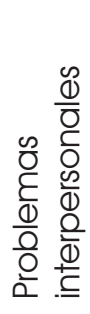 \\
\hline \multirow{12}{*}{ 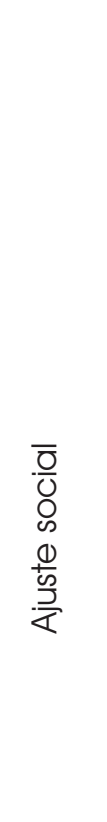 } & Reconciliación de la pareja &, 17 &, 17 &, 11 & $.27 *$ & $26^{*}$ & $25^{\star}$ &, 13 \\
\hline & Cambios en las condiciones de vida &, 02 & $23^{*}$ & $23^{*}$ & $21^{*}$ &, 16 &, 10 &, 08 \\
\hline & Discusiones con la pareja &, $24^{*}$ & 13 &, $32^{\star *}$ & $25^{*}$ &, $31^{*}$ &, $25^{\star}$ &, $22^{\star}$ \\
\hline & Divorcio de los padres &,$- 23^{*}$ & 17 &,$- 22^{\star}$ &,- 17 &,$- 24^{*}$ &,- 16 & $21^{*}$ \\
\hline & Cambio en las actividades sociales &,- 04 &, 20 & ,13 & $21^{\star}$ &, 12 &, 53 &, 10 \\
\hline & $\begin{array}{c}\text { Cambios en la forma o frecuencia de las } \\
\text { diversiones }\end{array}$ &,- 36 & 96 & 12 & $23^{*}$ &, 19 &, 51 &,- 00 \\
\hline & Cambio de horario o condiciones de trabajo &,- 40 &, 15 &,- 02 &, 10 &,- 12 & $23^{*}$ &, 20 \\
\hline & Enfermedad o incapacidad grave &, 14 &, 50 &, 88 & $33^{\star}$ &, 12 &,- 10 &, 25 \\
\hline & Problemas sexuales & ,75 &, 03 & 10 &,- 15 & $.22^{\star}$ &, 95 &, 15 \\
\hline & Embarazo &, 15 &, 11 &,- 23 &, 14 & $22^{\star}$ & 63 & 19 \\
\hline & Privación de la libertad &, 10 &,- 13 &,- 92 & $29^{*}$ &,- 80 &,- 62 &,- 52 \\
\hline & Total de sucesos estresantes & ,38 & $.25^{\star}$ &, 16 & , 12 & ,19 & , 19 &, 17 \\
\hline \multirow{5}{*}{ 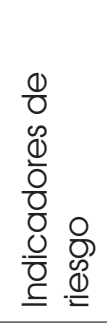 } & Dificultades de tipo amoroso &, $36^{\star}$ &, 13 &, $34^{\star \star}$ & $.31^{*}$ &, $39 * *$ &, $26^{\star}$ & $40^{\star}$ \\
\hline & Situación de violencia & $.21^{*}$ &, 94 & $20^{*}$ & , 18 &, $22^{*}$ &, $21^{*}$ &, $32^{\star}$ \\
\hline & Otras obligaciones además de estudiar & $23^{*}$ &,- 63 & 94 &, 12 &, 54 &, 52 & $25^{\star}$ \\
\hline & Asignaturas pendientes &,- 69 &,- 16 &,- 39 & $24^{*}$ &,- 84 & 40 &,- 10 \\
\hline & Otros & 19 & 10 & 10 & 14 & $25^{\star}$ & ,70 & $26^{*}$ \\
\hline
\end{tabular}

Se encontró que los sucesos de vida estresantes que correlacionaron en la mayoría de las dimensiones que evalúan la sintomatología depresiva, fueron las dificultades de tipo amoroso, discusiones con la pareja, experiencia de divorcio en la familia, experiencia de alguna situación de violencia y reconciliación de la pareja. Las afectaciones en el sueño y la fatiga presentaron frecuentes correlaciones con diferentes sucesos de vida estresantes. En el caso de la ideación suicida, se encontró que esta dimensión correlacionó de manera positiva y débil con los sucesos estresantes de discusiones en la pareja, así como, de reconciliación con la pareja, además, correlacionó de manera negativa con los cambios de horario o condiciones de trabajo (ver Tabla 7).

De este modo, los resultados permiten identificar los sucesos vitales estresantes y la sintomatología depresiva en una muestra de adolecentes de una zona rural, además de conocer que la sintomatología depresiva que 
se encuentra relacionada con dichos sucesos específicamente en esta población, como también fue posible identificar la diferencia en cuanto a la presencia de síntomas depresivos entre hombres y mujeres.

\section{DISCUSIÓN}

Los resultados de esta investigación permiten obtener información inicial sobre cuáles son los sucesos estresantes que experimentan los adolescentes, así como cuáles son los síntomas depresivos que presentan más y la relación existente entre ambas variables, así como las diferencias de género, contribuyendo a tener más evidencia de lo que ocurre con los jóvenes que viven y estudian en una comunidad rural en México. Los resultados se discutirán en función de cada uno de los objetivos.

En cuanto al objetivo de identificar la sintomatología depresiva y los sucesos vitales estresantes presentados con mayor frecuencia en los participantes, se encontró que los sucesos vitales estresantes que mencionaron los adolescentes con mayor frecuencia se relacionan con eventos cíclicos vinculados con el ámbito escolar en los que también participa de alguna forma la familia en consonancia con el ritmo que marcan las instituciones educativas, tales como las navidades, vacaciones o el inicio y cierre del ciclo escolar. Y es que tanto la Psicología del Desarrollo adolescente, como el análisis del ciclo vital de la familia, hacen referencia a las crisis previsibles e imprevisibles por las que los individuos y sus sistemas circundantes enfrentan (Semenova Moratto et al., 2015). Por lo que esas transiciones entre la escuela y casa implican para los adolescentes de este estudio, momentos críticos, o por lo menos, momentos percibidos como sucesos estresantes. También aparecen mencionados otros aspectos de tipo familiar e interpersonal como son los problemas familiares y la falta de comprensión por parte de los padres. Además, se mencionaron algunos indicadores de riesgo para la trayectoria académica como variables económicas, de salud y problemas de tipo amoroso (ej: "el enamoramiento que lleva a descuidar la escuela" según lo señalado en la Tabla 3).

En los adolescentes de este estudio, así como en población adulta de México (Acuña et al., 2012; Bruner, 1994) y de otros países (Holmes y Rahe, 1967), la muerte de un familiar cercano es uno de los sucesos de vida estresantes que ocupa uno de los primeros lugares como experiencia estresante, independientemente de la edad y de la nacionalidad. Para los adolescentes rurales ocupó el segundo lugar de entre los 43 sucesos estresantes evaluados. En esa misma línea, mencionan en sexto lugar la enfermedad de un pariente cercano. En sintonía, en el apartado de "otros problemas" los participantes resaltaron estos mismos temas al mencionar como sucesos estresantes "la muerte de una hermana", "el tener a mi hijo y después perderlo", "la muerte de mi abuelita", así como diversas situaciones de enfermedad en la familia.

Respecto al segundo objetivo del estudio de conocer las diferencias de género en cuanto a sintomatología depresiva y sucesos estresantes, los resultados de esta investigación sugieren la presencia de sintomatología depresiva en estudiantes de zonas rurales, observándose diferencias entre los hombres y las mujeres. Dichos resultados son coincidentes con otros estudios realizados en población urbana (Ramírez, 2010; Resende \& Ferrao, 2013) y con población que incluye tanto adolescentes de zona rural como urbana (Rivera-Heredia et al., 2013), en los cuales señalan la existencia de otras variables asociadas a la presencia de síntomas depresivos, tales como el estrés social, ansiedad, locus de control externo, abuso sexual, agresión escolar, migración y la existencia de conflictos dentro del sistema familiar. Es así que Sahril et al. (2019) señalaron que las mujeres tenían un 32\% de probabilidad de presentar una depresión severa en comparación con los hombres. Asimismo, Barra et al. (2019) encontraron diferencias en la presencia de sintomatología depresiva en cuanto a la existencia de dos eventos estresantes, los cuales son la conducta 
verbal agresiva de la madre y el padre. De igual forma, Bernaras et al. (2015) reportaron que la sintomatología depresiva en los hombres disminuye antes de los 12 años, mientras que, en las mujeres se registró un ligero aumento de dichos síntomas a esa misma edad.

Si bien la literatura científica señala que la presencia de estados depresivos elevados es más frecuente en las mujeres, es fundamental no dejar de prestar atención a la presencia de dicho padecimiento en los varones, tal como lo señalan González-Forteza et al. (2015) al indicar que la presencia de síntomas depresivos en los hombres puede tener como consecuencia repercusiones más serias, relacionadas con la conducta suicida, considerando que existe cierto estigma en cuanto a expresión de sentimientos y emociones en los hombres.

Respecto al tercer objetivo del estudio de analizar la relación existente entre las situaciones estresantes y la sintomatología depresiva, de acuerdo con los resultados de esta investigación, la presencia de situaciones vitales estresantes en los adolescentes se correlaciona con diversos tipos de síntomas depresivos, lo que coincide con lo reportado en los estudios previos, los cuales señalan la presencia de correlación entre dichas variables (Acosta-Hernández et al., 2014; Ferreira et al., 2012; Saleem et al., 2014; Veytia-López et al., 2012). Dichos estudios señalan específicamente la existencia de correlación en los sucesos estresantes de tipo familiar y escolar con la presencia de diversa sintomatología depresiva en adolescentes, informando que las situaciones estresantes que llegan a surgir en la vida de los adolescentes suelen propiciar la aparición o el desarrollo de dichos síntomas en los jóvenes, lo cual, a largo plazo, tiene consecuencias negativas, al afectar la calidad de vida y la salud mental de dicha población. Incluso, algunos de estos estudios (Cattelino et al., 2020; DiaconuGherasim y Măirean, 2020; Rahman et al., 2018) hacen énfasis en cómo la presencia de la sintomatología depresiva llega a causar afectación en el rendimiento académico de los adolescentes.
Como principal limitante del presente trabajo se encuentra el número de participantes y que estos pertenecen a una muestra de adolescentes escolarizados de una región rural, que es solo una porción del territorio mexicano, por lo que los resultados solo representan a este grupo de participantes. Por lo que un tema que podría explorarse en futuros estudios son las posibles diferencias en cuanto a sucesos vitales estresantes, indicadores de riesgo y sintomatología depresiva entre adolescentes escolarizados de población urbana con los provenientes de zonas rurales, además de replicar este tipo de estudios en diferentes países permitiendo con ello tener información transcultural. Dado que lo anteriormente señalado no son objetivos del presente estudio, constituyen áreas de oportunidad para próximos proyectos. Además de lo anteriormente mencionado sobre líneas de investigación para futuros proyectos sería importante realizar un estudio que también recupere datos relacionados con sintomatología ansiosa y con la regulación emocional.

Al hablar de sintomatología depresiva es importante recordar que su presencia tiene un impacto considerable en las vidas de las personas, sobre todo, en este caso se resalta la influencia que dichos síntomas tienen en cuanto a las temáticas relacionadas con el área educativa y en el desempeño escolar. El manejo de las pérdidas y de las enfermedades en la familia son temas clave que emergen como sugerencias para los programas de apoyo dirigidos hacia adolescentes escolarizados. Además de estos se sugiere incorporar el tema de manejo de las transiciones entre la escuela y la casa, y el cómo enfrentar los retos que se presentan en navidades, vacaciones o inicio o cierre del ciclo escolar. De igual manera el abordaje de los problemas de la vida amorosa de los adolescentes y los conflictos de pareja de sus padres emergieron como temas clave pues correlacionaron con la mayoría de las dimensiones del CESD-R (ver Tabla 7).

La prevención y la atención temprana dirigida a los adolescentes son especialmente 
relevantes dado que, tal como lo plantean González et al. (2018), Pascoe et al. (2020) y Shapero et al. (2014) la existencia de síntomas depresivos, tanto en la infancia como en la adolescencia, puede llegar a desencadenar episodios depresivos en la vida adulta, además de la presencia o desarrollo de otros problemas que afectan la salud mental y la calidad de vida, como son las adicciones o los comportamientos suicidas, entre otros. Como se mencionó anteriormente, por los resultados encontrados se sugiere también incorporar en el abordaje con los adolescentes aspectos de familia y pareja, tales como la interacción de los padres, sus conflictos y discusiones, el hecho de que se divorcien, y las propias dificultades en temas amorosos con su propia pareja, o con quien tienen la expectativa de tener un vínculo de ese tipo, pues todos ellos aparecieron en este estudio y son eventos estresantes a los que hay que poner especial atención en los programas de intervención preventiva y de atención para población de riesgo con sintomatología depresiva.

En conclusión, los resultados del presente estudio coinciden con lo reportado en la literatura científica, en relación a la presencia de síntomas depresivos en los adolescentes, que resultan más frecuentes en las mujeres. Por tanto, debido a la gravedad que conlleva este padecimiento y que además, la adolescencia es una etapa compleja del desarrollo, dentro de la cual se presentan cambios a nivel cognitivo, psicológico y físico, los cuales pueden llegar a desencadenar por sí solos estrés, se considera importante el diseño de estrategias y programas de prevención, que tengan como finalidad disminuir la presencia de dicha sintomatología en esta población. Además de fortalecer en los adolescentes sus recursos psicológicos y estrategias de afrontamiento (Pérez-Padilla et al., 2021) que les ayuden a sobrellevar los diversos obstáculos que se pueden llegar a manifestar a lo largo de la vida. Todo esto con el objetivo de mejorar su calidad de vida y su salud mental. De igual forma se considera, que una de las áreas que puede verse afectada por dicha problemática es la educativa. Por lo que el desarrollar acciones de prevención, podría ser una línea de futuras investigaciones, considerando que dichas estrategias sean de ayuda para disminuir el impacto de los síntomas depresivos en etapas de vida futuras de los adolescentes. Sobre todo al tomar en cuenta que el contexto escolar es un lugar idóneo para la implementación de este tipo de estrategias, tanto en instituciones educativas de zonas urbanas, como de aquellas más alejadas y regularmente con menor acceso a servicios como son las del área rural.

\section{- Conflicto de intereses.}

Los autores declaran no tener conflicto de intereses.

\section{REFERENCIAS}

Acosta-Hernández, M.E., Mancilla-Perano, T., Correa-Basurto, J., Saavedra-Vélez, M., Ramos-Morales, J., Cruz-Sanchéz, J. \& Durán-Niconott, S. (2014). Depresión en la infancia y adolescencia: enfermedad de nuestro tiempo. Archivos de Neurociencias, 16(1), 156-161. https:// www.medigraphic.com/pdfs/arcneu/ane2011/ane $111 \mathrm{e}$

Acuña, L., González, D. \& Bruner, C. (2012). La escala de reajuste social de Holmes y Rahe en México: Una revisión después de 16 años. Revista Mexicana de Psicología, 29(1), 16-32. http://www.redalyc.org/ $\mathrm{pdf} / 2430 / 243030189002$

Aliri, J., Muela, A., Gorostiaga, A., Balluerka, N., Aritzeta, A., \& Soroa, G. (2019). Stressful life events and depressive symptomatology among basque adolescents: The mediating role of attachment representations [Eventos de vida estresantes y sintomatología depresiva entre adolescentes vascos: el rol mediador de las representaciones del apego] Psychological Reports, 122(3). 789-808. https://journals.sagepub.com/ doi/abs/10.1177/0033294118771970 \#articleCitationDownloadContainer

Andrade-Palos, P., Betancourt, D., Vallejo, A., Celis, B. \& Rojas. R. (2012). Prácticas parentales y sintomatología depresiva en adolescentes. Salud Mental, 35(1), 29-36. http://www.redalyc.org/ pdf/582/58223290005. 
Askeland, K.G., Bøe, T., Breivik, K., La Greca, A. M., Sivertsen, B., \& Hysing, M. (2020). Life events and adolescent depressive symptoms: Protective factors associated with resilience [Eventos de vida y síntomas depresivos en adolescentes: factores protectores asociados con la resiliencia]. PLoS ONE, 15(6), Artículo e0234109. https://doi.org/10.1371/ journal.pone.0234109

Barcelata, B. \& Gómez-Maqueo, E. (2012). Fuentes de estrés y su influencia en la adaptación. En Claves del Pensamiento, 6(12), 31-48. http://www.scielo.org.mx/ pdf/enclav/v6n12/v6n12a2

Barra, S., Baharudin, R., Zulkefly, N., Yahya, A. \& Madon, Z. (2019). Understanding sex differences in depressive symptomatology among malaysian adolescents [Entendiendo diferencias en cuanto sexo en síntomas depresivos entre adolescentes de Malasia]. Recoletos Multidisciplinary Research Journal, 7, 63-79. https://www.researchgate.net/ publication/334393487 Understanding Sex Differences in Depressive Symptomatology among Malaysian Adolescents

Bernaras, E., Jaureguizar, J., Soroa, M., Ibabe, I. \& Cuevas, C. (2013). Evaluación de la sintomatología depresiva en el contexto escolar y variables asociadas. Anales de Psicología, 29(1), 131 . 140. http://www.scielo.isciii.es/ scielo. php? script $=$ sci arttext\&pid $=\quad$ S02129728201300010001

Bruner, C., Acuña, L., Gallardo, L., Atri, R., Hernández, A., Rodríguez, W., \& Robles, G. (1994). La escala de reajuste social (SRRS) de Holmes y Rahe en México. Revista Latinoamericana de Psicología, 26(2), 253-269. http://www.redalyc.org/ $\mathrm{pdf} / 805 / 80526204$

Buitrago-Matamoros, J. F., Pulido Archila, L. Y. \& Güichá-Duitama, A. M. (2017). Relación entre sintomatología depresiva y cohesión familiar en adolescentes de una institución educativa de Boyacá. Psicogente, 20(38), 296-307. https://doi. $\mathrm{org} / 10.17081 / \mathrm{psico} .20 .38 .2550$

Cattelino, E., Chirumbolo, A., Baiocco, R., Clanddri, E. \& Morelli, M. (2020). School
Achievement and Depressive Symptoms in Adolescence: The Role of Self-efficacy and Peer Relationships at School [Exito escolar y síntomas depresivos en adolescentes: el rol de la autoeficacia y relación con pares en la escuela]. Children Psychiatry Human Development, 52(4), 571-578. https:// doi.org/10.1007/s 10578-020-01043-z

Chapí, J. (2013). Características de la depresión. Revista digital EOS Perú, 1(1).59-65. http://www.scielosp.org/pdf/ rsap/v13n6/v13n6a04

Chow, C. M., Tan, C. C., \& Buhrmester, D. (2015). Interdependence of depressive symptoms, school involvement, and academic performance between adolescent friends: A dyadic analysis [Interdependencia de síntomas depresivos, participación escolar y desempeño académico entre compañeros adolescentes: un análisis diádico]. The British Journal of Educational Psychology, 85(3), 316-331. https://doi. org/10.1111/bjep.12075

Diaconu-Gherasim, L.R. \& Măirean, C. (2020). Depressive Symptoms and Academic Achievement: The Role of Adolescents' Perceptions of Teachers' and Peers' Behaviors [Síntomas depresivos y logro escolar: el rol de las percepciones de los maestros sobre los adolescentes y la conducta de los pares]. Journal of Research on Adolescence, 30, 471-486. https://doi.org/10.1111/iora.12538

Duggal, S., Carlson, E. A., Sroufe, L. A., \& Egeland, B. (2001). Depressive symptomato logy in childhood and adolescence. Development and Psychopathology, 13(1), 143-164. www. ncbi.nlm.nih.gov/pubmed/1 1346049

Ferreira, E., Granero, R., Noorian, Z., Romero, K., \& Doménech-Llaeria, E. (2012). Acontecimientos vitales y sintomatología depresiva en población adolescente. Revista de psicopatología y psicología clínica, 17(2), 123-136. http://www.infocop.es/view article. asp?id $=4468$

Fonseca-Pedrero, E., Paino, M., LemosGiraldez, G.\&Muñiz, J. (201 1). Prevalencia y características de la sintomatología depresiva en adolescentes no clínicos. 
Actas Españolas de Psiquiatría, 39(4), 217-25. https://dialnet.unirioja.es/ servlet/articulo? codigo $=3681331$

Frojd, S. A., Nissinen, E. S., Pelkonen, M. U. I., Marttunen, M. J., Koivisto, A.M. \& Kaltiala-Heino, R. (2008). Depression and school performance in middle adolescent boys and girls [Depresión y rendimiento escolar en niños y niñas en adolescentes de mediana edad]. Journal of Adolescence, $37(4), 485$ $498 . \quad$ https://doi.org/10.1016/i. adolescence.2007.08.006

Ghosh, T., Sijo, G. \& Suman, A. (2019). Prevalence and Correlates of Depressive Symptoms in Young Adolescents of Nepal [Prevalencia y correlación de síntomas depresivos en adolescentes jóvenes de Nepal]. The Clinical Neurologist International, 1, Artículo

1002. https://www.researchgate.net/ publication/339552661 Prevalence and Correlates of Depressive_Symptoms in Young Adolescents of Nepal/ link/5e58d57fa6fdccbeba080e77/ download

González-Forteza, C., Hermosillo de la Torre, A. E., Vacio-Muro, M. Á., Peralta, R. \& Wagner, F. (2015). Depresión en adolescentes. Un problema oculto para la Salud Pública y la práctica clínica. Boletín médico del Hospital Infantil de México, 72(2), 149-155. https://doi. org/10.1016/i.bmhimx.2015.05.006

González-Forteza, C., Jiménez-Tapia, J.A., Ramos-Lira, L. \& Wagner, F. (2008). Aplicación de la Escala de Depresión del Center of Epidemiological Studies en adolescentes de la Ciudad de México. Salud Pública de México, 50(4), 292 299. http://www.scielo.org.mx/scielo. php?script $=$ sci arttext\&pid $=$ S0036$\underline{36342008000400007 \& \operatorname{lng}=\mathrm{es} \&+\operatorname{lng}=}$ es

González, S., Pineda, A. \& Gaxiola, J. (2018). Adolescent Depression: Risk Factors and Social Support as a Protective Factor [Depresión adolescente: factores de riesgo y apoyo social como factor protector]. Universitas Psychologica, 17(3), 63-73. https://doi.org/10.11144/ Javeriana.upsy 17-3.dafr
Granrud, M. D., Steffenak, A. K. M., \& Theander, K. (2019). Gender differences in symptoms of depression among adolescents in Eastern Norway: Results from a cross-sectional study [Diferencias de género en sintomas depresivos entre adolescents del este de Norvega: resultados de un studio transveral]. Scandinavian Journal of Public Health, 47(2), 157165. https://journals.sagepub.com/doi/ abs/10.1177/1403494817715379\# articleCitationDownloadContainer

Humensky, J., Kuwabara, S. A., Fogel, J., Wells, C., Goodwin, B. \& Van Voorhees, B. W. (2010). Adolescents with depressive symptoms and their challenges with learning in school [Adolescentes con síntomas depresivos y sus retos con el aprendizaje en la escuela]. The Journal of school nursing: the official publication of the National Association of School Nurses, 26(5), 377-392. https://doi. org/10.1177/1059840510376515

Holmes, T. H., \& Rahe, R. H. (1967). The Social Readjustment Rating Scale. Journal of Psychosomatic Research, $11(2), 213-$ 218. https://doi.org/10.1016/00223999(67)90010-4

Jiménez-Tapia, A., Wagner, F., Rivera-Heredia, M.E. \& González-Forteza, C. (2015). Estudio de la depresión en estudiantes de la Ciudad de México y del Estado de Michoacán por medio de la versión revisada de la CES-D. Salud Mental, 38(2), 103-107. http://www.scielo.org.mx/scielo. php? script=sci arttext\&pid $=$ S0185$33252015000200004 \&$ lng =es\&tlng =es. Khesht-Masjedi, M. F., Shokrgozar, S., Abdollahi, E., Golshahi, M. \& SharifGhaziani, Z. (2017). Comparing depressive symptoms in teenage boys and girls [Comparando síntomas depresivos en chicos y chicas adolescentes]. Journal of Family Medicine and Primary Care, 6(4), 775-779. https://doi.org/10.4103/ifmpc. jfmpc 12917

Li, J. Y., Li, J., Liang, J. H., Qian, S., Jia, R. X., Wang, Y. Q. \& Xu, Y. (2019). Depressive Symptoms Among Children and Adolescents in China: A Systematic Review and Meta-Analysis [Síntomas depresivos entre niños y adolescents en China: una 
revision sistemática y meta-análisis]. Medical Science Monitor: International Medical Journal of Experimental and Clinical Research, 25, 7459-7470. https:// doi.org/10.12659/MSM.916774

López-López, J. A., Kwong, A., Washbrook, L., Tilling, K., Fazel, M. \& Pearson, R. (2021). Depressive symptoms and academic achievement in UK adolescents: a crosslagged analysis with genetic covariates [Síntomas depresivos y logro academic en adolescents de Reino Unido: un análisis cruzado con covariables géneticas]. Journal of Affective Disorders, 284, 104-113. https://doi.org/10.1016/i. jad.2021.01.091.

McMahon, G., Creaven, A.M. \& Gallagher, S. (2020). Stressful life events and adolescent well-being: The role of parent and peer relationships [Eventos estresantes y bienestar en adolescents: el rol de las relaciones con los padres y los pares]. Stress and Health, 36(3), 299310. https://doi.org/10.1002/smi.2923

Nunes, M., Borges, L. \&De Oliveira, A. (2017). Gender and Age-related Differences in Depressive Symptoms among Brazilian Children and Adolescents [Diferencias de género y edad relacionadas con síntomas depresivos entre niños y adolescentes brasileños]. Paideia, 27(68), 290-297. https://www.scielo.br/pdf/paideia/ v27n68/1982-4327-paideia-27-68-290

Organización Mundial de la Salud [OMS]. (2016). La depresión. http://www.who. int/mediacentre/factsheets/fs369/es/

Ospina-Ospina, F., Hinestrosa-Upegui, M., Paredes, M., Guzmán, Y. \& Granados, C. (2011). Síntomas de ansiedad y depresión en adolescentes escolarizados de 10 a 17 años en Chía, Colombia. Revista de Salud Pública, 13(6), 908-920. http://www. scielosp.org/pdf/rsap/v13n6/v13n6a04.

Pascoe, M., Hetrick, S. \& Parker, A. (2020). The impact of stress on students in secondary school and higher education [El impacto del estrés en estudiantes de escuela secundaria y niveles superiores de educación]. International Journal of Adolescence and Youth, 25(1), 104-112. https://doi.org/10.1080/02673843.201 9.1596823
Pérez-Padilla, E., Cervantes-Ramírez, V., Hijuelos-García, N., Pineda-Cortes, J., \& Salgado-Burgos, H. (2017). Prevalencia, causas y tratamiento de la depresión mayor. Revista Biomédica, 28(2), 73-98. https://doi.org/10.32776/revbiomed. v28i2.557

Pérez-Padilla, M. L., Rivera-Heredia, M. E., Esquivel-Martínez, C., Quevedo-Marín, M. C. \& Torres-Vázquez, V. (2021). Ser madre y estudiar la universidad. Recursos psicológicos, conductas problema y factores de riesgo. Revista de Psicología y Educación, 16(1), 1-17. https://doi. org/10.23923/rpye2021.01.198

Radloff, L. S. (1977). The CES-D Scale: A Self-Report Depression Scale for Research in the General Population [La escla CES-D: autoreporte de la escala de depression para población general]. Applied Psychological Measurement, 7 (3), 385-401. https://doi. org/10.1177/014662167700100306

Rahman, M., Todd, C., John, A., Tan, J., Kerr, M., Potter, R., Kennedy, J., Rice, F. \& Brophy, S. (2018). School achievement as a predictor of depression and self-harm in adolescence: Linked education and health record study [Logro escolar como predictor de la depression y la autolesión en adolescentes: estudio vinculado de clínica y educación]. The British Journal of Psychiatry, 212(4), 215-221. https:// doi.org/10.1192/bip.2017.69

Ramírez, F. (2010). Sintomatología depresiva en adolescentes mujeres: prevalencia y factores de relación interpersonal asociados. Revista Peruana de Epidemiología, 14(1), 65 7. https://www.redalyc.org/articulo. oa? id $=203119805009$

Resende, C. \& Ferrão, A. (2013). La presencia de síntomas depresivos en adolescentes en el último año de escolaridad. Pediatría Atención Primaria, 15 (58), 127-133. http://www.redalyc. $\mathrm{org} / \mathrm{pdf} / 3666 / 366638749004$

Reyes Ortega, M., Soto Hernández, A. L., Milla Kegel, J. G., García Ramírez, A., Hubard Vignau, L. Mendoza Sánchez, H., Mejía Garza, L. A., García Peña, M. C. y Wagner Echeagaray, F. A. (2003). Actualización de la Escala de Depresión 
del Centro de Estudios Epidemiológicos (CES-D). Estudio piloto en una muestra geriátrica mexicana. Salud Mental, 26(1),59-68. https://www.redalyc.org/ articulo.oa?id $=58212607$

Rivera, R., Arias-Gallegos, W. L., \& CahuanaCuentas, M. (2018). Perfil familiar de adolescentes con sintomatología depresiva en la ciudad de Arequipa, Perú. Revista Chilena de Neuro-psiquiatría, 56(2), 117 126. https://doi.org/10.4067/s0717. 92272018000200117

Rivera-Heredia, M. E., Martínez-Servín, L. G. \& Obregón-Velasco, N. (2013). Factores asociados con sintomatología depresiva en adolescentes. El rol de la familia migrante y los recursos individuales, familiares y sociales. Salud Mental, 36, 99-105. http://www.scielo.org.mx/pdf/ $\mathrm{sm} / \mathrm{v} 36 \mathrm{n} 2 / \mathrm{v} 36 \mathrm{n} 2 \mathrm{a} 4$

Rivera-Rivera, L., Rivera-Hernández, P., Pérez-Amescúa, M., Leyva-López, A. \& Castro, F. (2015). Factores individuales y familiares asociados con sintomatología depresiva en adolescentes de escuelas públicas de México. Salud Pública de México, 57(3). http://www.medigraphic. com/pdfs/salmen/sam-2013/sam 132d

Roberts, E. (1980). Reliability of the CES-D: Scale in different ethnic contexts [Fiabilida de la Escala CES-D: medición en diferentes contextos étnicos]. Psychiatry Research, 2(2), 125-134. https://www. sciencedirect.com/science/article/abs/ pii/0165178180900694?via\%3Dihub

Sahril, N., Yaacob, N. M., Ahmad, N. A., Abdullah, S., Naidu, B. M. \& Aris, T. (2019) Depression Severity and Its Associated Factors Among School-Going Adolescents in Malaysia [Gravedad de la depression y sus factores asociados entre adolescents escolarizados en Malasia]. Journal of Depression and Anxiety, 8, 350. https://doi.org/10.35248/2167. $\underline{1044.19 .8 .350}$

Saleem, S., Khalid, T. \& Mahmood, Z. (2014). Self-reported depressive symptomatology in adolescents: A psychometric study [Sintomatología depresiva reportada en adolescents: un estudio psicométrico]. FWU Journal of Social Sciences Summer, 8(1), 57-66. https://www.researchgate. net/publication/310596847 Self Reported Depressive Symptomatology in Adolescents A Psychometric Study

Shapero, B. G., Black, S. K., Liu, R. T., Klugman, J., Bender, R. E., Abramson, L. Y. \& Alloy, L. B. (2014). Stressful Life Events and Depression Symptoms: The Effect of Childhood Emotional Abuse on Stress Reactivity [Eventos de vida estresantes y síntomas depresivos: el efecto del abuso emocional en la infancia sobre la reactividad del estrés]. Journal of Clinical Psychology, 70(3), 209-223. https://doi. org/10.1002/iclp.22011

Semenova Moratto, N., Zapata Posada, J. J. y Messager, T. (2015). Conceptualización de ciclo vital familiar: una mirada a la producción durante el periodo comprendido entre los años 2002 a 2015. CES Psicología, 8(2),103121. https://www.redalyc.org/articulo. oa? id $=423542417006$

Stikkelbroek, Y., Bodden, D. H. M., Kleinian, M., Reijnders, M. \& Van Baar, A. L. (2016). Adolescent Depression and Negative Life Events, the Mediating Role of Cognitive Emotion Regulation [Depresión adolescente y eventos de vida negativos, el rol mediador de la regulación emocional cognitiva]. PLoS ONE, 11 (8) Articulo e0161062, 1-16. https://doi. org/10.1371/iournal.pone.0161062

Suárez, M. (2010). La importancia del análisis de los acontecimientos vitales estresantes en la práctica clínica. Revista Médica La Paz, 16(2), 58-62. http:// www.scielo.org.bo/pdf/rmcmlp/v16n2/ v16n2 a 10

Tafur-Orahulio, Y. M. (2020). Sintomatología ansiosa-depresiva en relación con el funcionamiento familiar en un grupo de adolescentes de Lima Norte. CASUS, 5(1), 18-25. https://casus.ucss.edu.pe/ index.php/casus/article/view/228/167

Veytia-López, M., Guadarrama Guadarrama, R., Márquez-Mendoza, O. \& Fajardo Gómez, R. (2016). Mindfulness y síntomas de depresión en adolescentes mexicanos estudiantes de bachillerato. Actualidades en Psicología, 30(121), 39-48, 02586444. https://www.redalyc.org/articulo. oa? id $=1332 / 133248870006$ 
Veytia, M., González, N., Andrade-Palos, P. \& Oudhof, H. (2012). Depresión en adolescentes: El papel de los sucesos vitales estresantes. Salud mental, (35), 37-43. http://www.scielo.org.mx/pdf/sm/ v35n 1/v35nla6.pdfhttp://www.scielo. org. mx/pdf/sm/v35nl/v35nlab

Villegas-Pantoja, M. Alonso-Castillo, M. Alonso-Castillo, B. \& Guzmán, F. (2014). Eventos estresantes y la relación con el consumo de alcohol y tabaco en adolescentes. Ciencia y enfermería, 20(1), 35-46. http://www.scielo.cl/pdf/ cienf/v20n1/art 04
Yue, L., Dajun, Z., Yinghao, L. \& Tianquiang, H. (2016). Metaanalysis of the Relationship between Life Events and Depression in Adolescents [Metaanalisis de las relacuines entre eventos de vida estresantes y depression en adolescentes]. Journal of Pediatric Care, 2(1), 1-13. https://pediatrics.imedpub. $\mathrm{com} / \mathrm{metaanalysis-of-the-relationship-}$ between-life-events-and-depression-inadolescents 